\title{
Computational Modeling of Gas-Surface Interactions for High-Enthalpy Reacting Flows
}

\author{
Abhilasha Anna*, Hicham Alkandry ${ }^{\dagger}$ and Iain D. Boyd ${ }^{\ddagger}$ \\ Department of Aerospace Engineering, University of Michigan, Ann Arbor, MI, 48109
}

\begin{abstract}
The aerothermal heating of a Thermal Protection System (TPS) is significantly affected by the physical and chemical interactions that occur between the planetary entry vehicle surface and the hypersonic atmospheric gas. To study these processes, a gas-surface interaction model is used in the present numerical analysis that accounts for surface catalytic reactions and surface participating reactions. The study examines the effects of gas-surface interactions for graphite exposed to high enthalpy reacting nitrogen flow. The processes analyzed are the catalytic recombination of nitrogen atoms to molecules at the surface and the carbon nitridation reaction where nitrogen atoms react with the surface carbon to form gaseous CN. The results obtained using a computational fluid dynamic (CFD) code are assessed using data from experimental tests conducted in a $30 \mathrm{~kW}$ Inductively Coupled Plasma (ICP) Torch Facility at the University of Vermont. The species concentration gradient in the boundary layer and heat flux transferred to the surface are strongly affected by the surface reactions. The rate of carbon mass removal due to carbon nitridation is also calculated and compared to the measured value.
\end{abstract}

\section{Nomenclature}

$D_{k} \quad$ Diffusion coefficient of species $k\left[\mathrm{~m}^{2} / \mathrm{s}\right]$

$E_{a d} \quad$ Energy barrier for adsorption, $[\mathrm{J} / \mathrm{mol}]$

$E_{E R} \quad$ Energy barrier for Eley-Rideal recombination, $[\mathrm{J} / \mathrm{mol}]$

$J_{s p} \quad$ Species diffusion flux $\left[\mathrm{kg} / \mathrm{m}^{2} / \mathrm{s}\right]$

$k_{b} \quad$ Backward reaction rate, units vary

$k_{f} \quad$ Forward reaction rate, units vary

$K \quad$ Total number of species [dimensionless number]

$\dot{m} \quad$ Mass blowing rate due to surface reactions, $\left[\mathrm{kg} / \mathrm{m}^{2} / \mathrm{s}\right]$

$M_{k} \quad$ Molar weight of species $k[\mathrm{~kg} / \mathrm{mol}]$

$N_{n b} \quad$ Number of bulk species [dimensionless number]

$N_{s p} \quad$ Number of species in the mixture [dimensionless number]

$N_{R} \quad$ Number of surface reactions [dimensionless number]

$R_{u} \quad$ Universal gas constant $[\mathrm{J} / \mathrm{mol} / \mathrm{K}]$

$\bar{\nu}_{k} \quad$ Thermal speed of gas phase species $k[\mathrm{~m} / \mathrm{s}]$

$\nu_{k i}^{\prime} \quad$ Reactant stoichiometric coefficient for species $k$ in reaction $i$ [dimensionless number]

$\nu_{k i}^{\prime \prime} \quad$ Product stoichiometric coefficient for species $k$ in reaction $i$ [dimensionless number]

$\dot{w} \quad$ Production rate $\left[\right.$ moles $\left./ \mathrm{m}^{2} / \mathrm{s}\right]$

$h_{s p} \quad$ Species enthalpy $[\mathrm{J} / \mathrm{kg}]$

$k_{B} \quad$ Boltzmann constant

$q \quad$ Total heat flux $\left[\mathrm{W} / \mathrm{m}^{2}\right]$

$q_{\text {conv }} \quad$ Convective heat flux $\left[\mathrm{W} / \mathrm{m}^{2}\right]$

$q_{\text {diff }} \quad$ Diffusive heat flux $\left[\mathrm{W} / \mathrm{m}^{2}\right]$

$S_{0} \quad$ Sticking coefficient [dimensionless number]

* Graduate Student, Student Member AIAA.

$\dagger$ Research Associate, Member AIAA.

${ }^{\ddagger}$ James E. Knott Professor, Fellow AIAA. 


$\begin{array}{ll}T & \text { Translational-rotational temperature }[\mathrm{K}] \\ Y & \text { Mass fraction [dimensionless number] } \\ \gamma & \text { Reaction efficiency [dimensionless number] } \\ \kappa & \text { Thermal conductivity }[\mathrm{W} / \mathrm{m} / \mathrm{K}] \\ \rho & \text { Density }\left[\mathrm{kg} / \mathrm{m}^{3}\right] \\ \sigma & \text { Stefan-Boltzmann constant }\left[\mathrm{W} / \mathrm{m}^{2} / \mathrm{K}^{-4}\right] \\ \text { subscripts } & \\ b & \text { bulk phase } \\ \infty & \text { reference freestream conditions } \\ \text { sp } & \text { species value } \\ t r & \text { translational-rotational energy mode } \\ v e & \text { vibrational-electronic energy mode } \\ w & \text { wall value }\end{array}$

\section{Introduction}

A ATMOSPHeric entry probes and hypersonic vehicles experience heat loads during an entry into the A atmosphere of Earth or any other planet. The heat loads are due to the dissipation of kinetic energy of a high-speed flow that cause very high temperature on the vehicle surface. Therefore, such vehicles use a Thermal Protection System (TPS) for protection from aerodynamic heating. TPS is a single point of failure system as the prolonged exposure to high temperature can cause the TPS materials to fail. ${ }^{1,2}$ The need to design a reliable TPS necessitates good understanding of the physical and chemical processes that determine the aerothermal heating environment.

Depending on the heat load encountered during hypersonic flight, an ablative or non-ablative TPS may be used. $^{2}$ Non-ablative or reusable materials (e.g., ceramic tiles used on the Space Shuttle) are used where the re-entry conditions are mild while ablative TPS materials (e.g., heat shield for the Apollo mission) are used where high heating rates are generated. There is no change in mass or properties of the non-ablative material whereas ablative TPS materials accommodate high heating rates and heat loads through phase change and mass loss. Ablative TPS has been used for most planetary entry probes and high velocity Earth atmosphere re-entry vehicles. The net heat flux ${ }^{3}$ experienced by an ablative TPS surface may consist of (1) convective heating as a result of gas particle collisions and their interactions with the surface, and (2) radiative heating as a result of radiation from excited particles in the flow. Catalycity of an ablative TPS material and surface-participating reactions that lead to surface recession are key factors that impact the heating of the vehicle surface. Very high temperatures in the boundary layer may cause the molecular species to dissociate. If the heated TPS material acts as a catalyst and dissociated atoms diffuse to the surface, it may cause recombination of dissociated boundary layer species which increases the convective heating to the surface. Thus, a less catalytic surface is desirable to minimize this additional heating. Also, when the vehicle surface is heated, the surface material may chemically react with the boundary layer gases leading to surface recession as a result of surface material consumption. These chemical reactions can be endothermic (vaporization, sublimation) or exothermic (oxidation, nitration) and will affect the net heating to the surface. Therefore, detailed studies of these interactions that occur between the surface and the atmosphere gas are required for the accurate prediction of aerothermal heating of the vehicle TPS and in characterizing TPS materials.

To study the effects of gas-surface interaction processes, the entry flight environment considered in the present study represents the post shock subsonic high-enthalpy gas flow. The characterization of a TPS material requires it to be tested in an experimental facility that can create conditions similar to real entry flight conditions. Reproducing these conditions in existing ground facilities is both challenging and expensive. ${ }^{4,5}$ Therefore, a partial simulation of the flight conditions that involves subsonic high-enthalpy flow for the purpose of this study is considered. Computational models can be used for accurately predicting the aerothermal environment of the vehicle TPS during (re)-entry, but can be used to perform such analysis only after they have been validated to accurately predict the flow in the test facility and are validated with experimental results from the facility. The ground test facility of interest here is the Inductively Coupled Plasma (ICP) Torch Facility ${ }^{6,7}$ at the University of Vermont. The experimental tests conducted in this facility are used for the evaluation of the present computational results. The high-enthalpy nitrogen flow 
over a graphite sample placed in the test chamber of the ICP torch facility is simulated computationally. Hot nitrogen plasma is generated in the ICP torch and flows into the test chamber of the facility through the ICP torch exit. The exit of the ICP torch is the inlet to the test chamber of the facility flow where the sample is tested. Previous studies ${ }^{8,9}$ examined the effects of surface catalysis and the nature of the flowfield in the ICP torch facility for graphite exposed to high-enthalpy nitrogen flow. In Ref. 8, the surface catalysis effects were accounted for by using a simplified catalytic atom recombination model where the recombination of nitrogen atoms to molecules due to catalysis was investigated. It was shown that the temperature in the boundary layer is not affected by surface catalysis whereas it strongly affects the boundary layer gradients of species concentration, and heat transfer to the surface. It was also shown that the flow in the ICP torch facility is in thermochemical nonequilibrium. A comparative analysis was also performed to evaluate the effects of uniform and a non-uniform flow profiles at the ICP torch exit (i.e., the test chamber inlet) on the flow around the graphite sample. It was shown that the uniform profile provides better agreement with the experimental measurements of translational temperature whereas the non-uniform inlet profile provides better agreement with the experimental measurements of number density. It was also concluded in the study that the non-uniform inlet profile does not significantly affect the solution. The current study is an extension to the work presented in Refs. 8 and 9. A more detailed gas-surface interaction model is used that accounts for surface catalytic reactions as well as the surface participating reactions. Therefore, in addition to nitrogen atom recombination to nitrogen molecules, the reaction between atomic nitrogen and surface carbon to form gaseous $\mathrm{CN}$ is taken into account. The objective of this research study is to assess the effect of different gas-surface interaction models and processes on the species concentration and temperature gradients near the material surface, and on the heat transfer to the material surface. The carbon mass removal due to carbon nitridation reaction is also computed and compared to the measured value.

This paper is presented in four sections. The first section describes the numerical method followed by a description of the experimental facility and its set up where the graphite sample is tested in the high-enthalpy nitrogen flow. The details of the numerical set up for the simulations are provided in the second section. The results are presented in the next section where a discussion of the effects of gas-surface interactions for various surface reactions on the numerical solution is provided along with the comparison of computed results with measured experimental data for translational temperature and relative nitrogen atom number density in the flow in front of the test article. The paper ends with some conclusions drawn from these comparisons and suggestions for future work.

\section{Technical Approach}

\section{A. Numerical Method}

The numerical simulations in this study are conducted using the Michigan Aerothermodynamic NavierStokes computational fluid dynamics (CFD) code LeMANS, ${ }^{10-12}$ developed at the University of Michigan. It is a general purpose, parallel, three-dimensional code that solves the laminar Navier Stokes equations including chemical and thermal nonequilibrium effects on unstructured computational grids. The flow is modeled assuming the continuum approximation is valid. The set of partial differential equations are solved using a finite-volume method on unstructured grids. The inviscid fluxes across cell faces are discretized using a modified form of the Steger-Warming Flux Vector Splitting (FVS) scheme ${ }^{13}$ which is less dissipative and produces better results in boundary layers. The viscous terms are calculated using the values of properties at the cell centers and at the nodes. Time integration is performed using a point implicit or a line implicit method. LeMANS is parallelized using METIS ${ }^{14}$ that partitions the computational grid between the processors and Message Passage Interface (MPI) protocol to communicate information between processors. It is assumed that the translational and rotational energy modes of all species can be described by a single temperatures $\mathrm{T}_{t r}$, while the vibrational energy mode and electron energy of all species can be described by a single temperature $\mathrm{T}_{v e}$. LeMANS can simulate two-dimensional/axisymmetric flows using any mixture of quadrilaterals and triangles, and three-dimensional flows using any mixture of hexahedra, tetrahedral, prisms and pyramids. All the axisymmetric simulations are performed in this study using computational grids that contain quadrilateral elements.

\section{Species surface boundary conditions}

The boundary conditions at the wall are defined by a gas-surface interaction model ${ }^{15-17}$ implemented 
in LeMANS. It is a general finite rate surface chemistry (FRSC) model that can be used to investigate the effects of surface catalysis as well as surface participating reactions. The FRSC model developed by Marschall and Maclean ${ }^{15,16}$ is implemented in LeMANS by Alkandry et. al. ${ }^{17}$ The model can simulate the chemical interaction between the hypersonic gas and surface of the vehicle during planetary entry. In our prior work, a simplified binary catalytic atom recombination model was used to study the effects of surface catalysis. It could only be applied to a binary gaseous mixture of atoms and molecules. The FRSC model can be applied to multiple gaseous species and can account for different surface reactions such as particle adsorption/desorption, the recombination of an atom of the gas with an atom adsorbed on the wall [Eley-Rideal (E-R) reaction], recombination of two adsorbed atoms at the wall [Langmuir-Hinshelwood (L-H) reaction] and reactions leading to surface recession (e.g. carbon nitridation, oxidation).

For a system with $K$ species and $N_{R}$ surface reactions, the general form of the surface reaction $i$ can be expressed as,

$$
\sum_{k=1}^{K} \nu_{k i}^{\prime} A_{k} \rightleftharpoons \sum_{k=1}^{K} \nu_{k i}^{\prime \prime} A_{k}
$$

The net production rate $\dot{w}_{k}$ of species $k$ is the sum of the production rates from all surface reactions given by,

$$
\dot{w}_{k}=\sum_{i=1}^{N_{R}} \dot{w_{k i}}
$$

where the reaction-specific production rate $\dot{w_{k i}}$ is given by the expression shown in Eq. 3 .

$$
\dot{w_{k i}}=\left(\nu_{k i}^{\prime \prime}-\nu_{k i}^{\prime}\right)\left(k_{f i} \prod_{k=1}^{K} X_{k}^{\nu_{k i}^{\prime}}-k_{b i} \prod_{k=1}^{K} X_{k}^{\nu_{k i}^{\prime \prime}}\right)
$$

where $k_{f i}$ and $k_{b i}$ are the forward and backward reaction rates for reaction $i$, respectively, and $X_{k}$ is the concentration of species $k$ at the surface. The forward reaction rate for each surface reaction type is specified using a kinetic-based formulation. The FRSC model can account for competing finite-rate processes under a given set of experimental conditions and provides an effective reaction efficiency for a gas-phase reactant consumed in a surface reaction process. For this study, the FRSC model is used to simulate a constant reaction efficiency by using the appropriate choice of reaction types and parameters. The gas-surface interaction processes studied are the recombination of nitrogen atoms to molecules at the surface due to catalysis, and the carbon nitridation reaction where nitrogen atoms react with the surface carbon to form gaseous CN. The surface reaction types considered are adsorption and Eley-Rideal (E-R) recombination. The E-R mechanism involves the reaction of a gas-phase species with an adsorbed species to form a gas-phase product. The surface reaction for an adsorption process for a particle A can be represented by,

$$
A+(s) \rightarrow A(s)
$$

where (s) is an empty active site and $\mathrm{A}(\mathrm{s})$ is an adsorbed particle. The forward reaction rate for an adsorption process is expressed by the following :

$$
k_{f}=\left[\frac{\bar{\nu}_{A}}{4 \phi_{s}^{\nu_{s}}}\right] S_{0} \exp \left(-\frac{E_{a d}}{R_{u} T}\right)
$$

The surface reaction for an Eley-Rideal (E-R) recombination of a particle A with an adsorbed particle B(s) can be represented by,

$$
A+B(s) \rightarrow A B+(s)
$$


The forward reaction rate for an Eley-Rideal (E-R) recombination process is expressed by the following :

$$
k_{f}=\left[\frac{\bar{\nu}_{A}}{4 \phi_{s}^{\nu_{s}}}\right] \gamma_{0} \exp \left(-\frac{E_{E R}}{R_{u} T}\right)
$$

The thermal velocity $\bar{\nu}_{A}$ of species $A$ is given by the following expression:

$$
\bar{\nu}_{A}=\sqrt{\frac{8 R u T}{\pi M_{A}}}
$$

The backward reaction rate is zero as both the thermal desorption and dissociation with a partial adsorption process (shown by Eq. 9) are not considered in this study.

$$
\begin{aligned}
& A(s) \rightarrow A+(s): \text { Thermal desorption } \\
& A B+(s) \rightarrow A+B(s): \text { Dissociation with partial adsorption }
\end{aligned}
$$

The E-R reaction can also be used to represent a process where a gas phase species impinges on the surface and reacts with the surface. An example is shown in Eq. 10, where the gas phase species A impinges on the surface and reacts with the bulk phase species $B_{b}$ on the surface.

$$
A+(s)+B_{b} \rightarrow A B+(s)
$$

The species mass fraction at the wall is calculated by balancing the mass flux of the relevant species taking the consumption/production at the wall into account as shown in Eq. 11,

$$
-\left.\rho_{w} D_{k} \frac{\partial Y_{k}}{\partial n}\right|_{w}+\rho_{w} v_{w} Y_{k, w}=M_{k} \dot{w}_{k}
$$

where $\rho_{w} v_{w}$ is the mass blowing rate $\dot{m}_{b}$ at the surface due to surface reactions (e.g. oxidation, nitridation and sublimation). It is given by the following expression:

$$
\dot{m}_{b}=\rho_{w} v_{w}=-\sum_{k=1}^{N_{n b}} M_{k} \dot{w}_{k}
$$

\section{Inlet and outlet boundary conditions}

The flow in the case of an ICP torch test facility is subsonic in nature. Therefore, the inlet and outlet are specified for subsonic flow conditions. ${ }^{18}$ For the subsonic inlet boundary, all the variables are directly specified. For the subsonic outlet boundary condition, a constant pressure boundary condition is implemented in which static pressure is specified at the outlet. The velocity and density variables are solved using zeroth order extrapolation. The specified outlet pressure is used to compute the temperature variable using the equation of state.

\section{Thermochemical model}

For the analysis where only catalytic nitrogen atom recombination at the surface is considered, the gas mixture is composed of atomic and molecular nitrogen using the following dissociation-recombination reaction:

$$
\begin{aligned}
& N_{2}+M \rightleftharpoons 2 N+M \\
& M=N, N_{2}
\end{aligned}
$$

The mixture transport properties for this case are calculated using Gupta's mixing rule ${ }^{19}$ with species viscosities and thermal conductivities calculated using collision cross section data. The mixture transport 
properties for this case are also calculated using Wilke's semi-empirical mixing rule ${ }^{20}$ with Blottner's curve fits $^{21}$ and Eucken's relation ${ }^{22}$ and no difference is observed between the results from two mixing rule models. The results calculated using Gupta's mixing rule for this case are presented in this paper.

For the analysis where both catalytic nitrogen atom recombination and carbon nitridation reaction at the surface is considered, the gas mixture is considered composed of atomic nitrogen, molecular nitrogen, atomic carbon and the $\mathrm{CN}$ molecule and uses the following dissociation-recombination reactions:

$$
\begin{aligned}
& N_{2}+M \rightleftharpoons 2 N+M \\
& C N+M \rightleftharpoons C+N+M \\
& M=N, N_{2}, C, C N
\end{aligned}
$$

The mixture transport properties for this case are modeled using Wilke's semi-empirical mixing rule ${ }^{20}$ with species viscosities calculated using Blottner's model ${ }^{21}$ and the species thermal conductivities are determined using Eucken's relation. ${ }^{22}$ The mixture transport properties are not calculated using Gupta's mixing rule ${ }^{19}$ for this case because of the unavailability of the collision cross section data for CN species reactions. The species mass production rates are modeled using a finite-rate chemistry model. ${ }^{23}$ In this study, dissociation reactions are modeled using Park's two-temperature model wherein it is assumed that the rotational and translational energy modes of all species can be described by a single temperature $T_{t r}$ and the vibrational energy mode of all species and the electron energy can be described by a single temperature $\mathrm{T}_{v e}{ }^{24}$

\section{Heat flux at the wall}

Heat transfer to the surface is composed of convective heat flux $\mathrm{q}_{c o n v}$ and heat flux $\mathrm{q}_{\text {diff }}$ due to diffusion of species to the surface. The convective heat flux is composed of convection $\mathrm{q}_{c o n v, t r}$ due to the translational and rotational modes and $\mathrm{q}_{c o n v, v e}$ due to the vibrational mode. The convective heat flux is modeled according to Fourier's law and species diffusion heat fluxes are modeled using a modified form of Fick's law. ${ }^{25}$ They are given by the following expressions:

$$
\begin{aligned}
& q=q_{\text {conv }}+q_{d i f f} \\
& q_{c o n v, t r, v e}=-\kappa_{\text {tr }, v e} \frac{\partial T_{t r, v e}}{\partial n} \\
& q_{d i f f}=\sum_{i=1}^{N_{s p}} h_{i} J_{i}
\end{aligned}
$$

where $\kappa_{t r, v e}$ is the thermal conductivity for each energy mode, $\mathrm{h}_{i}$ is the species enthalpy and $\mathrm{J}_{i}$ is the normal species diffusion flux. The wall is assumed isothermal and is set to the prescribed wall temperature $\mathrm{T}_{w}$.

\section{B. Experimental Setup}

Assessment of the simulations is performed using experimental tests that were conducted in the $30 \mathrm{~kW}$ Inductively Coupled Plasma (ICP) Torch Facility ${ }^{7,26}$ at the University of Vermont. It is designed to test scaled material samples in high enthalpy gas flows for simulation of planetary entry and Earth atmosphere reentry trajectory heating conditions. It is configured for operation with subsonic flow to simulate post shock conditions of high enthalpy flight for a stagnation point geometry. For this study, experimental results from POCO graphite grade DFP2 samples tested in the nitrogen plasma stream are used. The experimental tests included measurement of the nitrogen atom number density and translational temperature in the reacting boundary layer above the graphite surface using a two-photon laser induced fluorescence (LIF) technique. Total mass removal rate from the sample was also measured.

The test article is a $19.05 \mathrm{~mm}$ diameter graphite sample mounted in the test chamber at a distance of $116 \mathrm{~mm}$ from the ICP torch exit. Figure 1 shows a photograph of the graphite sample during exposure to the nitrogen plasma in the test chamber of the ICP Torch Facility. Hot nitrogen plasma flows out (shown by green arrows in Figure 1) through the quartz tube of the ICP torch at a mass flow rate of $0.8 \mathrm{~g} / \mathrm{s}$. The quartz tube has a $36 \mathrm{~mm}$ internal diameter and is located in the gas injection assembly of the ICP Torch

Facility. The exit of the quartz tube of the ICP torch is the inlet to the test chamber of the facility where the sample is tested. The nitrogen gas enters from the bottom at room temperature into the quartz confinement 


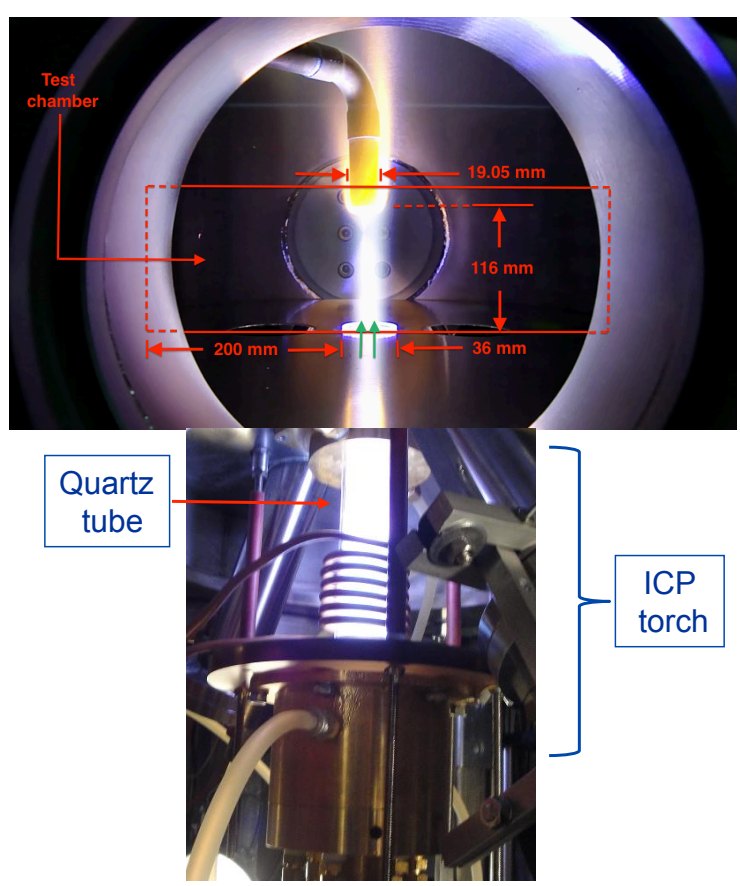

Figure 1. Experimental set up with graphite sample in nitrogen plasma in the test chamber of the ICP torch facility (section in box is the portion simulated using the CFD code LeMANS) (Source: Prof. D.G. Fletcher ${ }^{6}$ ).

tube where hot nitrogen plasma is generated through an induced RF magnetic field created by a helical load coil. The hot nitrogen plasma then flows out of the quartz tube from the top into the test chamber of the ICP facility where the sample is tested.

\section{Numerical Setup}

The facility inlet conditions (i.e., the ICP Torch exit conditions ) and the graphite sample wall temperature used for this study are based on the experimental set up and are provided in Table 1.

Table 1. Freestream and wall boundary conditions.

\begin{tabular}{|l|l|l|l|}
\hline $\begin{array}{l}\text { Mass flow rate } \\
(\mathrm{kg} / \mathrm{s})\end{array}$ & $\begin{array}{l}\text { Temperature, } \\
T_{\infty}[\mathrm{K}]\end{array}$ & $\begin{array}{l}\text { Pressure } \\
{[\mathrm{kPa}]}\end{array}$ & $\begin{array}{l}\text { Wall Temperature, } \\
T_{\text {wall }}[\mathrm{K}]\end{array}$ \\
\hline \hline $0.8 \times 10^{-3}$ & 7000 & 12.5 & 1590 \\
\hline
\end{tabular}

The equilibrium composition of nitrogen gas mixture at the quartz tube exit for the given temperature and pressure ${ }^{26}$ are calculated for the uniform inlet using the NASA program Chemical Equilibrium with Applications $^{27}$ (CEA). The results of this computation show a significant level of nitrogen dissociation. The prominent species present in the gas mixture are molecular and atomic nitrogen with mole fraction of 0.11 and 0.89 respectively.

The boundary conditions set for the simulation are shown in Fig. 2. The test chamber wall is set as a non-catalytic wall ${ }^{28,29}$ with a wall temperature of $300 \mathrm{~K}$. The gas-surface interaction model is applied only to the test article surface.

The gas-surface interaction processes studied are the recombination of nitrogen atoms to molecules at the surface due to catalysis, and carbon nitridation where nitrogen atoms react with the surface carbon to form gaseous CN. Carbon nitridation is studied as sample mass loss is observed in the experiment and results for the surface recession are reported in the work by Lutz et al. ${ }^{26}$ Therefore, two sets of surface reactions are taken into account. The first set is the surface reaction (shown in Eq. 16) that accounts only for the nitrogen 


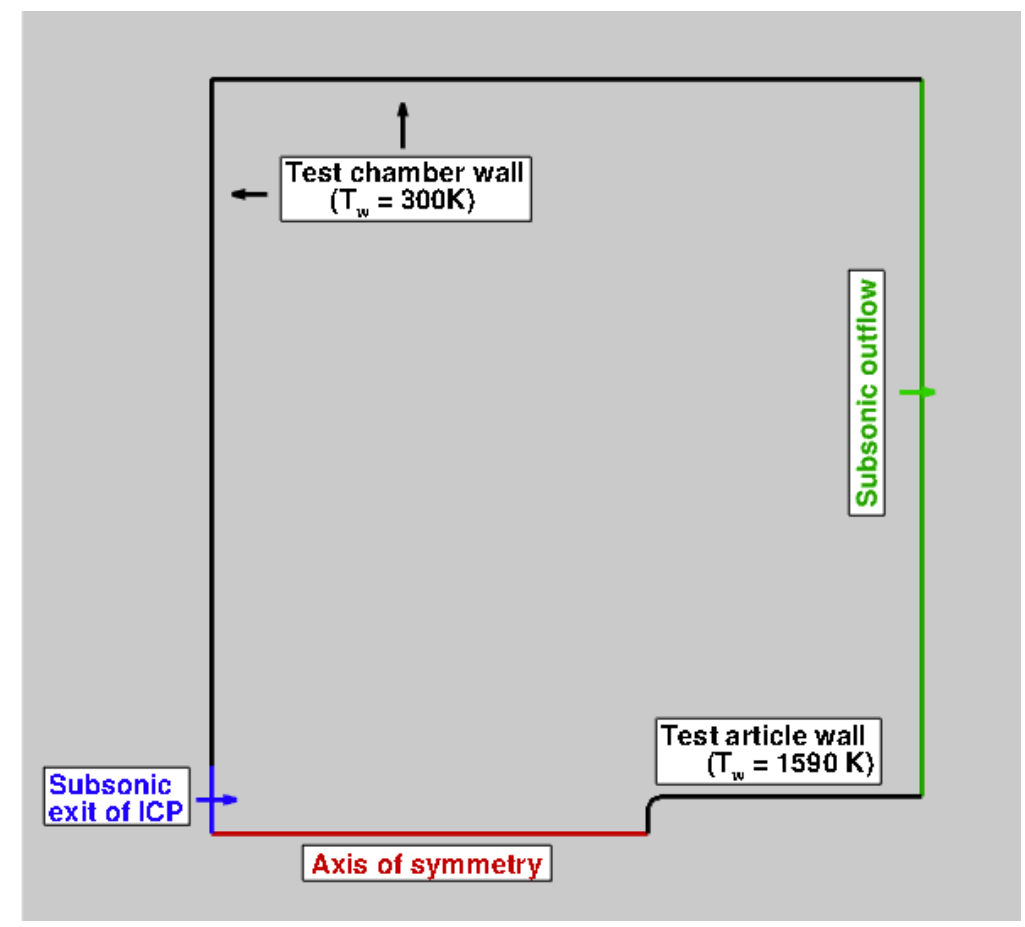

Figure 2. Boundary conditions

atom recombination on the wall due to surface catalysis.

$$
\begin{aligned}
& N+(s) \rightarrow N(s): \text { Adsorption }\left(E_{a d}=0 \mathrm{~J} / \mathrm{mol}\right) \\
& N+N(s) \rightarrow N_{2}+(s) \text { : Eley-Rideal recombination }\left(E_{E R}=0 \mathrm{~J} / \mathrm{mol}\right)
\end{aligned}
$$

The second set (shown in Eq. 17) takes into account the nitrogen atom recombination on the wall due to surface catalysis along with the carbon nitridation reaction where the carbon from the surface reacts with the impinging nitrogen atoms. The Eley-Rideal recombination reaction is used to represent the process of carbon nitridation.

$$
\begin{aligned}
& N+(s) \rightarrow N(s) \text { : Adsorption }\left(E_{a d}=0 \mathrm{~J} / \mathrm{mol}\right) \\
& N+N(s) \rightarrow N_{2}+(s) \text { : Eley-Rideal recombination }\left(E_{a d}=0 \mathrm{~J} / \mathrm{mol}\right) \\
& N+(s)+C_{b} \rightarrow C N+(s): \text { Eley-Rideal recombination }\left(E_{a d}=0 \mathrm{~J} / \mathrm{mol}\right)
\end{aligned}
$$

All the test cases are investigated for constant reaction efficiency $\gamma_{0}$. To achieve a constant net reaction efficiency, both the reaction efficiency $\gamma_{0}$ and sticking coefficient $S_{0}$ are set to the same value. The net reaction efficiency follows the relationship shown in Eq. 18.

$$
\gamma=\frac{2 S_{0} \gamma_{0}}{S_{0}+\gamma_{0}}
$$

The reaction efficiency $\gamma_{0}$ for surface catalysis, also referred to as catalytic efficiency of nitrogen atoms, is denoted by $\gamma_{N}$. It is defined as the ratio of the flux of nitrogen atoms that recombine on the surface to form nitrogen molecules to the total flux of nitrogen atoms that impinge on the surface. The reaction efficiency for carbon nitridation, also referred to as carbon nitridation efficiency, is denoted by $\gamma_{C N}$. It is defined as the ratio of nitrogen atoms reaching the surface and combining with surface carbon atoms to the ratio of the total flux of nitrogen atoms that impinge on the surface. It is assumed that all the carbon mass loss occurs due to the carbon nitridation reaction. The test cases considered in this study to determine the effects of gas-surface interaction processes are shown in Table 2. 
Table 2. Test cases.

\begin{tabular}{|l|l|l|}
\hline Case & Catalytic efficiency $\gamma_{N}$ & $\begin{array}{l}\text { Carbon nitridation efficiency } \\
\gamma_{C N}\end{array}$ \\
\hline \hline Case 1 & 0 & 0 \\
\hline Case 2 & 0.07 & 0 \\
\hline Case 3 & 0.07 & 0.005 \\
\hline Case 4 & 0.07 & 0.3 \\
\hline Case 5 & 1 & 0 \\
\hline
\end{tabular}

The catalytic efficiency $\gamma_{N}$ is set to zero for a non-catalytic wall and is set to 1 for a fully-catalytic wall. The partially catalytic wall condition of $\gamma_{N}=0.07$ is based on an experimentally determined value ${ }^{30}$ for pure carbon. The value $\gamma_{C N}=0.005$ for Case 3 is set based on a value determined by Driver and Maclean. ${ }^{31}$ It is obtained from a comparison between data from arc jet tests performed for Phenolic Impregnated Carbon Ablator (PICA) in nitrogen and results from computational simulations. The nitridation efficiency of $\gamma_{C N}$ produces good predictions of both heat transfer and recession rate when both reactions i.e. carbon nitridation and nitrogen atom recombination to nitrogen molecules due to catalysis, are included in the computations. ${ }^{31}$ The value $\gamma_{C N}=0.3$ for Case 4 is set based on a value determined by Park and Bogdanoff. ${ }^{32}$ It is measured in a shock tube by passing highly dissociated nitrogen over a grid of tungsten wire coated with carbon. It should be noted that $\gamma_{C N}$ is dependent on the type of carbon used as well as the experimental conditions.

Case 1 represents a wall where no surface chemistry is accounted for and is treated as non-catalytic. The surface chemistry for Cases 2 and 5 is defined by the reactions shown in Eq. 16 and for Cases 3 and 4, it is defined by Eq. 17.

An axisymmetric configuration is used for all simulations. The grid is generated using the commercial mesh generation software Pointwise. ${ }^{33}$ Grid independence is achieved for a flow in thermal equilibrium using a grid with 50,000 quadrilateral cells. This grid is used for all the test cases studied.

\section{Results}

In this section, the results of the study that used the CFD code LeMANS to determine the effects of gas-surface interaction processes on a graphite sample exposed to a high enthalpy and low pressure flow are provided. The flow physics model chosen for all test cases is thermochemical nonequilibrium flow. Thermochemical nonequilibrium (TCNE) means that the flow is both in vibrational and chemical nonequilibrium where both vibrational relaxation and finite rate of chemical relaxation are considered. The translational and rotational modes are assumed to be equilibrated.

The main calculated parameters analyzed are translational temperature, relative nitrogen atom density, and surface heat flux. The comparisons between the numerical results and experimental LIF measurements are presented for translational temperature and relative nitrogen atom density in the test sample boundary layer along the stagnation streamline and in the radial direction at an axial distance of $1.52 \mathrm{~mm}$ from the test article surface. The relative nitrogen atom density is calculated by scaling the numerical profile obtained from LeMANS for Case 5 (which is expected to provide the best agreement with the measurements) to agree with the measurement at the largest distance from the test sample and then the scaled factor is used to obtain the relative axial stagnation line and radial profiles for all five simulation conditions. The stagnation line boundary layer results are shown in Fig. 3, with translational temperature in Fig. 3(a) and the relative nitrogen atom density in Fig. 3(b). The radial profile results for translational temperature and relative nitrogen atom number density are shown in Figs. 4(a) and 5(a), respectively. The region where the radial measurements are taken is shown in the temperature and relative nitrogen atom density contours for Case 5 in Figs. 4(b) and 5(b), respectively. The experimental temperature and relative nitrogen atom density values have uncertainties of about $\pm 500 \mathrm{~K}$ and $\pm 25 \%$, respectively. ${ }^{6}$

The surface chemistry does not significantly affect the temperature in the boundary layer, both axially and radially, as shown in the temperature profiles in Figs. 3(a) and 4(a), respectively. The effect of surface chemistry on nitrogen atom density in the boundary layer can be seen in Figs. 3(b) and 5(a), respectively. It can be seen in Fig. 3(b) that nitrogen atom density increases in the boundary layer for Case 1 whereas it 


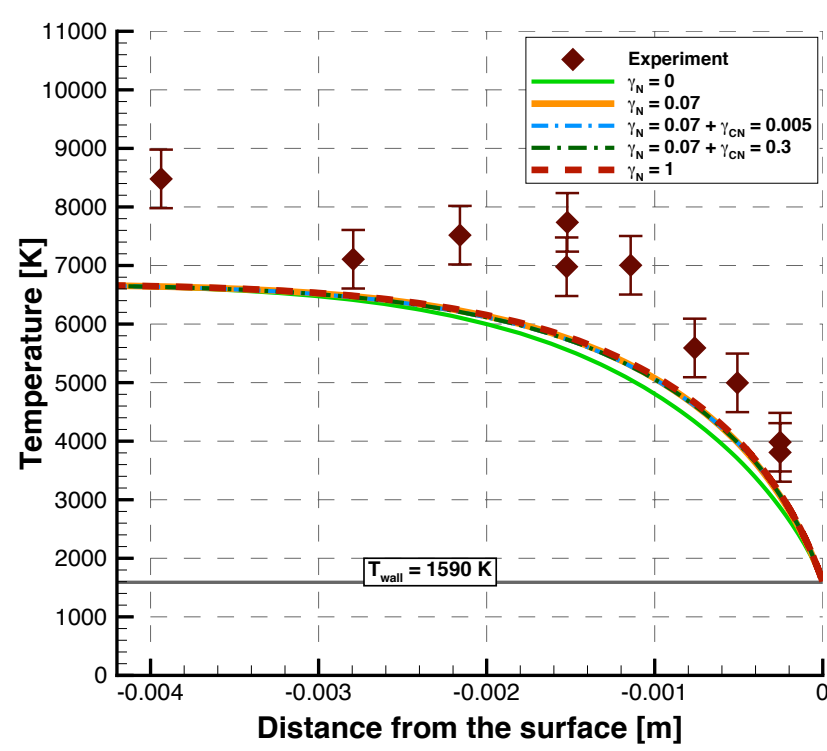

(a) Translational temperature

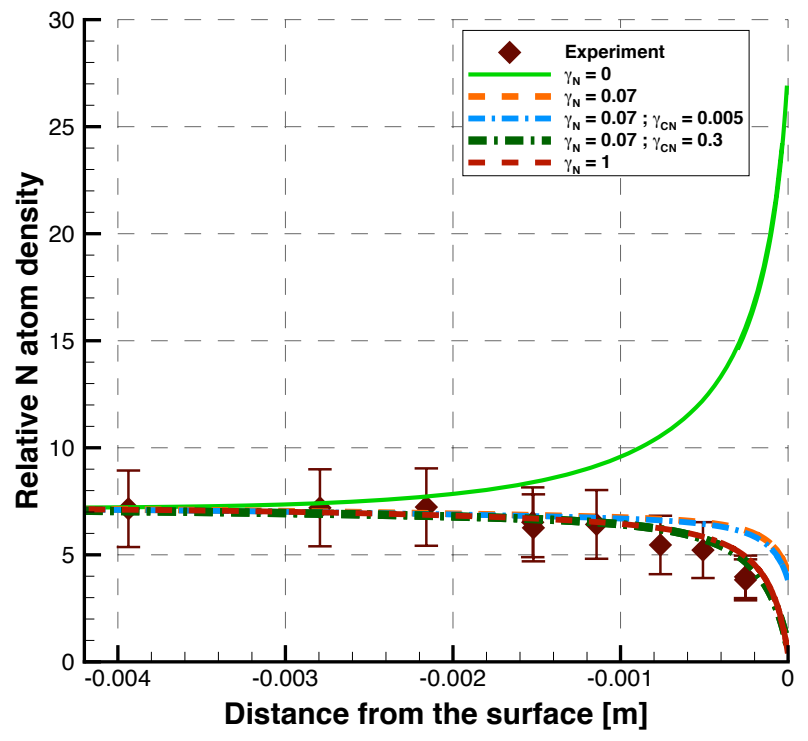

(b) Relative N-atom density

Figure 3. Comparison of translational temperature and relative $\mathrm{N}$-atom density along the stagnation line between the computational and experimental data.

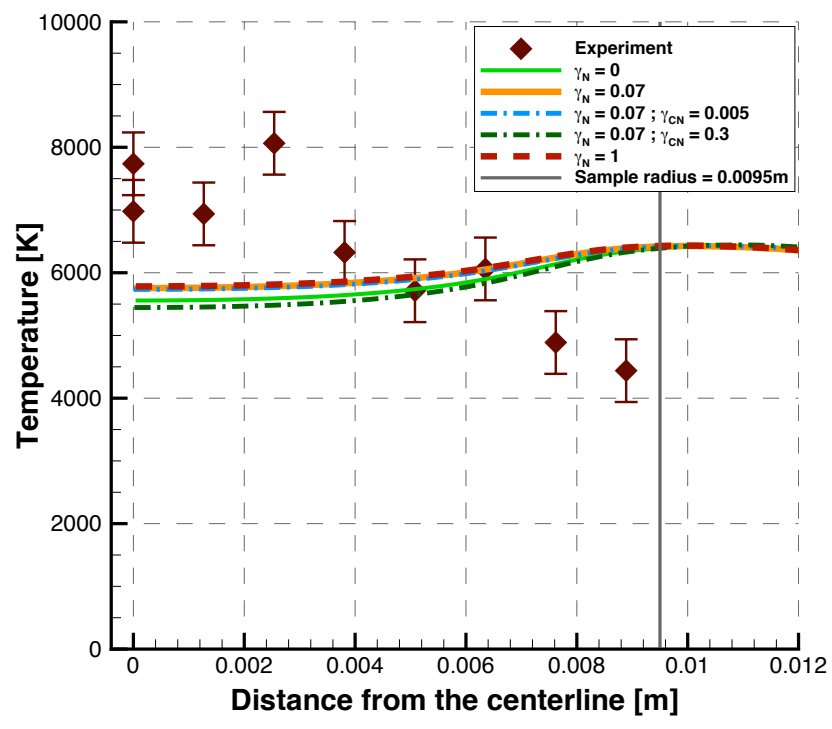

(a) Translational temperature

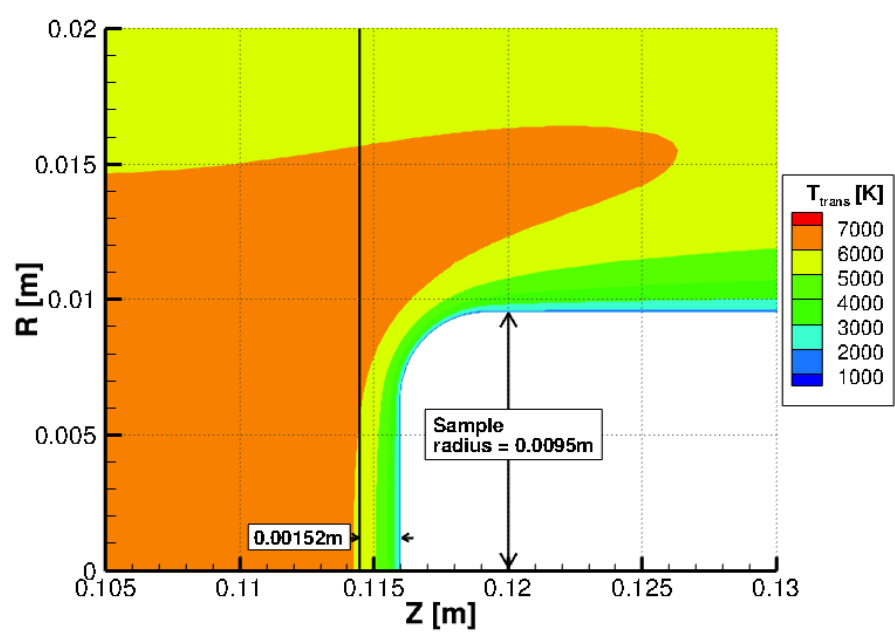

(b) Translational temperature contours

Figure 4. (a) Comparison of translational temperature between the computational and experimental data in the radial direction at a distance of $1.52 \mathrm{~mm}$ from the stagnation point. (b) Translational temperature contours for Case $5\left(\gamma_{N}=1\right)$ showing the region of radial measurement. 


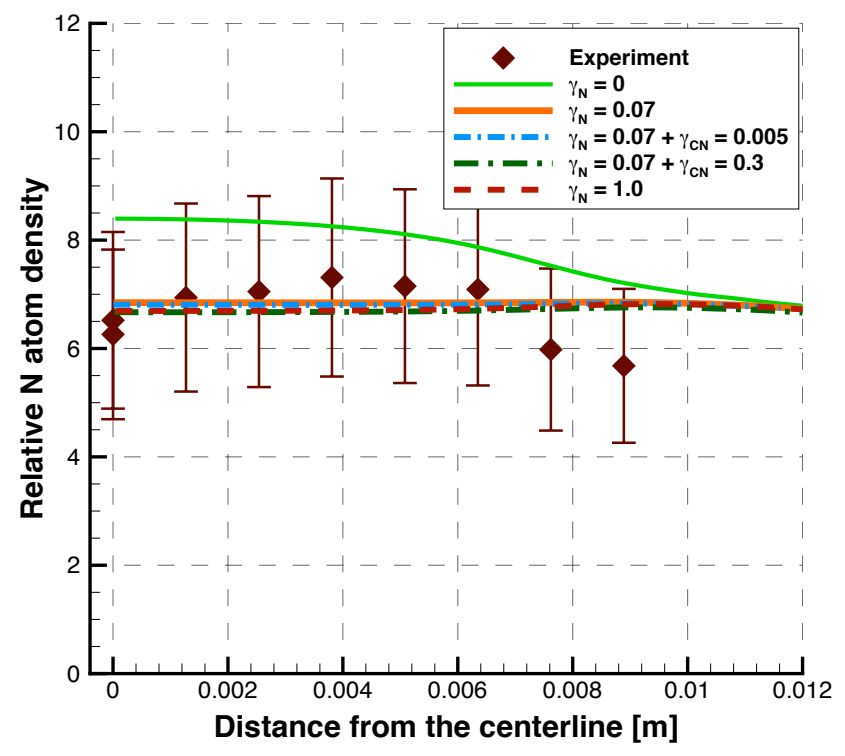

(a) Relative N-atom density

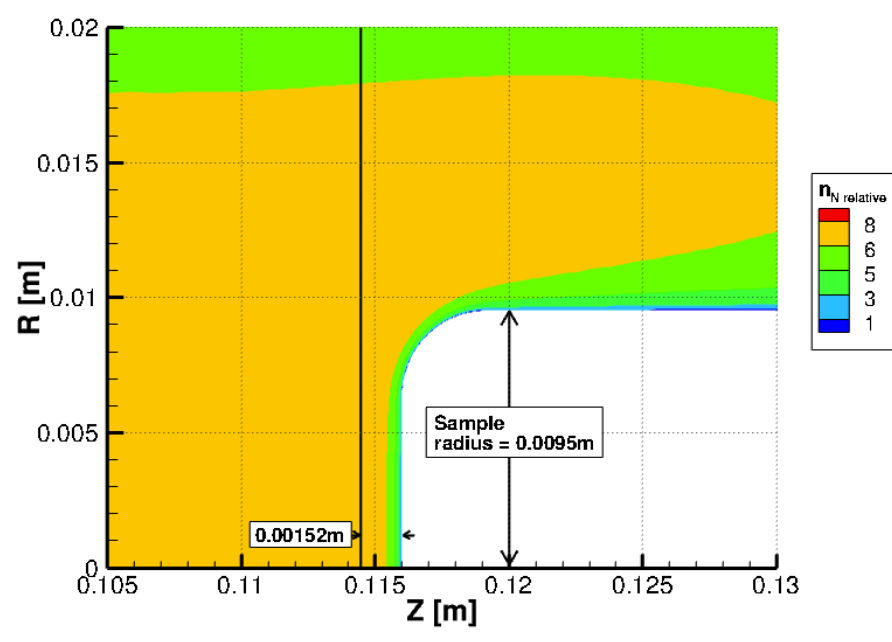

(b) Relative N-atom density contours

Figure 5. (a) Comparison of relative N-atom density between the computational and experimental data in the radial direction at a distance of $1.52 \mathrm{~mm}$ from the stagnation point. (b) Relative N-atom density contours for Case $5\left(\gamma_{N}=1\right)$ showing the region of radial measurement.

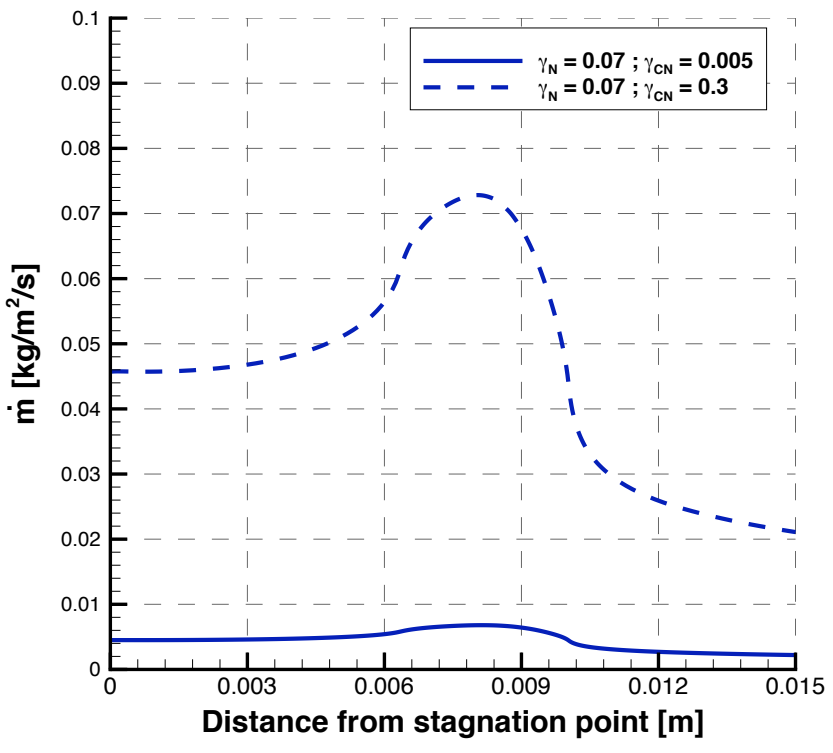

(a) Mass removal rate

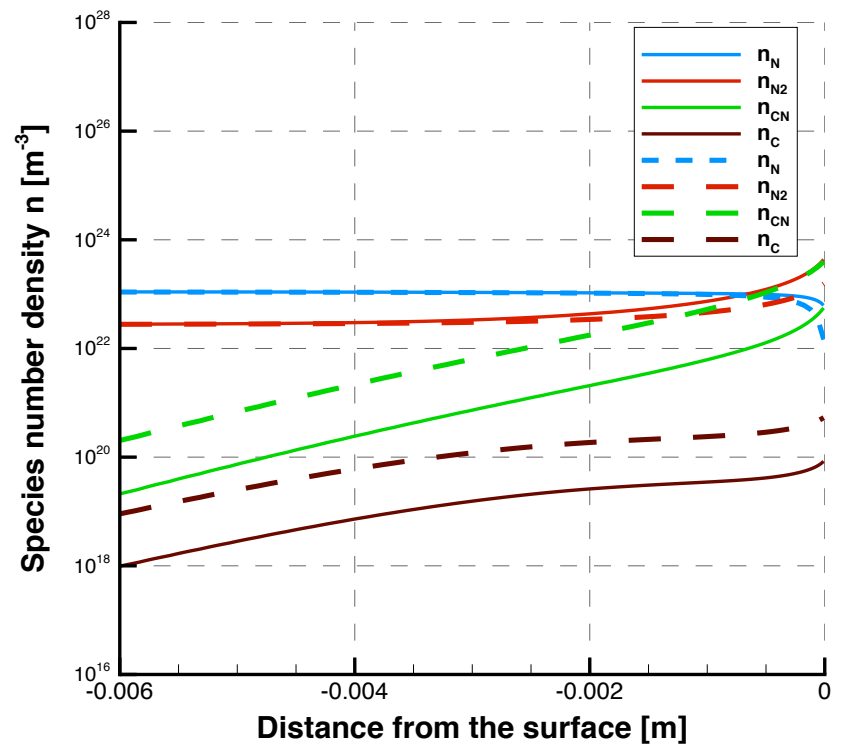

(b) Species number density

Figure 6. (a) Mass removal rate due to carbon nitridation (b) Species number density along the stagnation line (Case 3: solid line and Case 4: dashed line). 
is depleted for all other cases. The nitrogen atom loss is due to surface catalysis i.e. catalytic recombination of nitrogen atoms to molecules for Cases 2 and 5. As expected, the nitrogen atom density for a partially catalytic surface, i.e. for $\gamma=0.07$, lies between the profiles for non-catalytic and fully catalytic walls. The nitrogen atom loss seen for Cases 3 and 4 is both due to surface catalysis as well as carbon nitridation. The nitrogen atom removal for Case 3, i.e. for $\gamma_{N}=0.07$ and $\gamma_{C N}=0.005$, is largely due to surface catalysis with negligible carbon nitridation whereas the nitrogen atom loss for Case 4, i.e. for $\gamma_{N}=0.07$ and $\gamma_{C N}=$ 0.3 , is largely due to carbon nitridation. The nitrogen atom density for all cases except Case 1 show good agreement with the experimental measurements.

A similar trend is observed in the radial profiles in Fig. 5(a), with nitrogen atom density highest for a Case 1 and lowest for Cases 4 and 5. The depletion of nitrogen atoms (Fig. 3(b) and 5(a)) observed in the computational results for a catalytic wall is explained by the strong effect of surface catalysis as well as carbon nitridation. The nitrogen atom loss for Case 5 where it is only to due to surface catalysis is comparable to Case 4 where it is largely due to carbon nitridation in addition to the partially catalytic wall. The mass removal rate $\left(\dot{m}_{b}\right)$ calculated by Eq. 12 is shown in Fig. 6(a) for Cases 3 and 4. Although Cases 3 and 4 agree well with the experimental results, the carbon mass removal for these cases is much higher than measured experimentally. The experimentally determined value of mass loss rate of carbon is $0.33 \mathrm{mg} / \mathrm{s}$. The computationally calculated value for mass loss rate of carbon for Case 3 is $3.7 \mathrm{mg} / \mathrm{s}$ and for Case 4 is 37 $\mathrm{mg} / \mathrm{s}$. The mass loss rate is calculated as shown in Eq. 19.

$$
\text { mass loss rate }=\int \dot{m_{b}} d A
$$

where $\dot{m}_{b}$ is the mass removal rate for each surface element and $d A$ is the surface area of each element.

The computed high ablation rate could be explained by a combined effect of various mechanisms. The higher mass removal rate suggests that the carbon nitridation efficiency may be smaller. In the experimental tests, as the real test continues, the shape of the shoulder changes as the mass is removed and it is likely that the mass removal rate also decreases. The changing shape of the sample is not yet accounted for in the computational analysis. Also, it is possible that some of the carbon species that are blown into the flow through carbon nitridation may be re-absorbed onto the surface and therefore reduce the net mass removal rate. The reactions for this process are shown in Eq. 20 .

$$
\begin{aligned}
& C N \rightarrow C+N: \text { Dissociation } \\
& C+(s) \rightarrow C(s)
\end{aligned}
$$

As seen in Eq. 17, only the reaction of nitrogen atoms with surface carbon is taken into account in the present simulations. It is also possible for the $\mathrm{CN}$ molecule to react further with nitrogen atoms to form nitrogen molecules and the carbon atoms may thus be re-absorbed onto the surface. The reactions for this process are shown in Eq. 21. These mechanisms will be added to the computational model in future studies.

$$
\begin{aligned}
& N+(s)+C_{b} \rightarrow C N+(s): \text { Eley-Rideal recombination } \\
& C N+N \rightarrow N_{2}+C \\
& C+(s) \rightarrow C(s)
\end{aligned}
$$

The degree of nitrogen atom flux to the surface may also affect the amount of mass removed. An assumption of chemical equilibrium of the nitrogen gas mixture at the exit of the quartz tube is used in the present computations. The equilibrium gas mixture composition is probably more dissociated than the composition for a chemically reacting flow with finite rate chemistry. Lower nitrogen atom flux to the surface would result in a lower mass removal rate. Therefore, a sensitivity analysis will be performed on the ICP torch exit chemical composition in future studies.

The mass removal rate could also be affected by the net heat transfer to the surface. The wall in the computations is assumed isothermal and is set to a constant temperature of $1590 \mathrm{~K}$. If a radiative equilibrium wall boundary condition is assumed, the calculated surface temperature is much higher and is shown in Fig. 7. A higher surface temperature would result in reduced convective heating. The radiative wall boundary condition will be used in future studies for a more accurate analysis. The surface temperature 
profile shown in Fig. 7 is calculated using Eq. 22 with emissivity $\epsilon$ for graphite set to $0.9 .{ }^{34}$ Also, the heat conduction within the sample is not included in the computation, which if accounted for, may reduce the surface temperature.

$$
q_{c o n v}+q_{d i f f}=\sigma \epsilon T^{4}
$$

The species number densities in the boundary layer for Cases 3 and 4 are shown in Fig. 6(b). It can be seen that the $\mathrm{CN}$ molecule concentration in the boundary layer increases and the nitrogen molecule concentration decreases as the nitridation efficiency is increased.

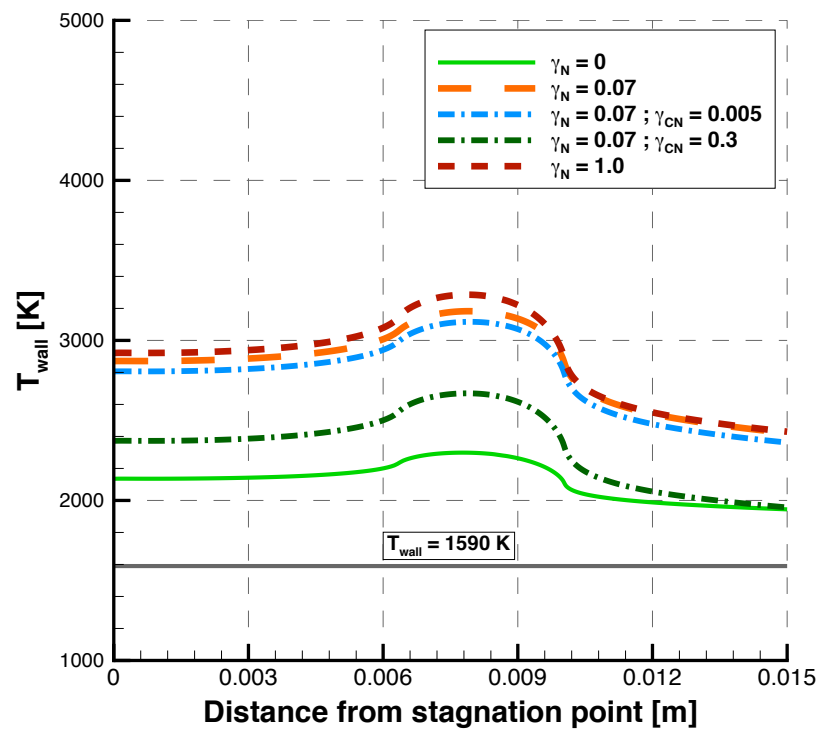

Figure 7. Comparison of wall temperature for different test conditions listed in Table 2.

Figure 8 presents the calculated wall heat fluxes for all cases investigated. The total heat flux and the diffusive heat flux are shown in Figs. 8(a) and 8(b), respectively. As expected, there is an increase in the total heat flux for all the cases with surface reactions as compared to the non-catalytic wall. There is a significant increase in the total heat flux for a catalytic wall as compared to the non-catalytic wall. This increase is explained by the contribution from diffusive heat flux for the cases with surface reactions which is zero for a non-catalytic wall as shown in Fig. 8(b). All the surface reactions considered are exothermic. The diffusive heat flux for Cases 3 and 4 that include carbon nitridation in addition to surface catalysis is less than the diffusive heat flux obtained for Cases 2 and 5 that include the surface catalysis alone. The reason for this is attributed to the different heats of formation of the nitrogen and $\mathrm{CN}$ molecules. The heat of formation for nitrogen is $940 \mathrm{~kJ} / \mathrm{mol}$ and for $\mathrm{CN}$, it is $590 \mathrm{~kJ} / \mathrm{mol}$. Therefore, the lower diffusive heat flux for carbon nitridation can be explained by a lower heat of formation of the $\mathrm{CN}$ molecule.

\section{Conclusion}

The purpose of this research was to study the effects of gas-surface interaction processes on the numerical simulation of a graphite sample exposed to a subsonic high-enthalpy nitrogen flow. The gas-surface interaction processes studied were the recombination of nitrogen atoms to molecules at the surface due to catalysis and the carbon nitridation reaction where nitrogen atoms react with the surface carbon to form gaseous CN. A finite-rate surface chemistry (FRSC) model that can account for different surface reactions such as particle adsorption/desorption, the recombination of an atom of the gas with an atom adsorbed on the wall [Eley-Rideal (E-R) reaction] as well as reactions leading to surface recession, was used to study these processes. The numerical simulation was performed using the CFD code LeMANS. The computations were assessed using experimental tests conducted in the $30 \mathrm{~kW}$ Inductively Coupled Plasma (ICP) Torch Facility $^{7,26}$ at the University of Vermont. The effects of surface reactions were evaluated on the flow field 


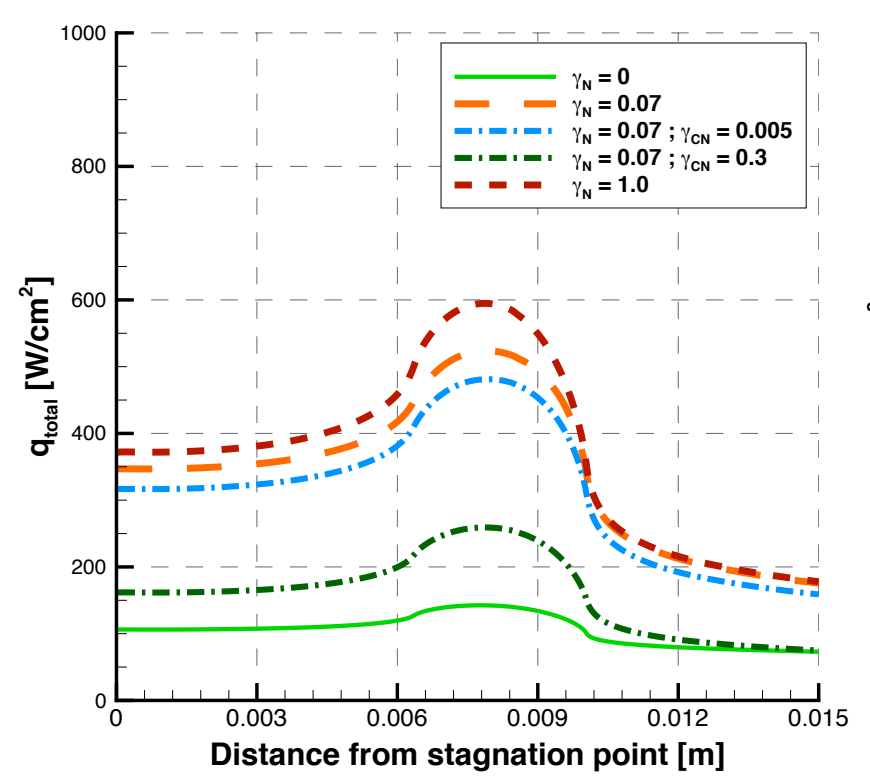

(a) Total heat flux

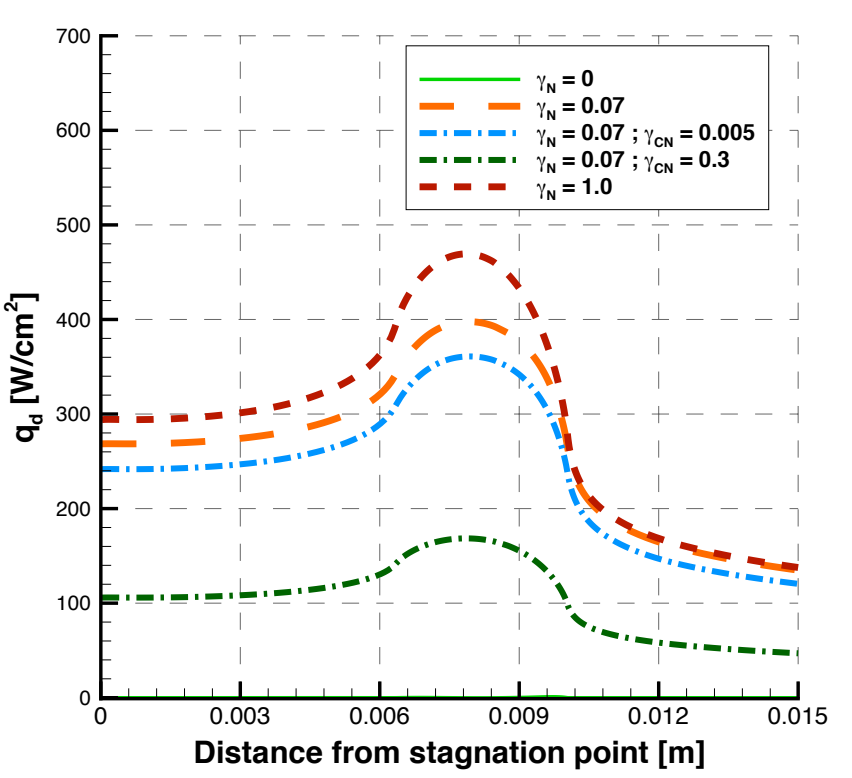

(b) Diffusive heat flux

Figure 8. Comparison of wall heat flux between the computational results for different test conditions listed in Table 2.

around the graphite sample and its surface properties. The carbon mass removal rate as a result of the carbon nitridation reaction was also computed and compared to the measured value. The results from the simulations showed that the temperature in the boundary layer is not significantly affected by different surface reactions whereas the nitrogen atom density decreased in the boundary layer when surface chemistry was included. The nitrogen atom number density profiles were relatively insensitive to the surface chemistry parameters, and all four cases showed good agreement with the experimental measurements. The carbon mass removal rate computed was higher than the measured experimental value. An increase in the heat flux transferred to the surface with chemical reactions was observed as compared to the non-catalytic wall.

This work is considered as the continuation of the study of gas-surface interaction processes and development of more accurate methods to model these processes. Different elementary surface reactions (such as $\mathrm{CN}+\mathrm{N} \rightarrow N_{2}+\mathrm{C}, \mathrm{C}+(\mathrm{s}) \rightarrow \mathrm{C}(\mathrm{s})$ ) other than the ones included in this study will be accounted for in future calculations. A sensitivity analysis on the ICP torch exit chemical composition will be performed to evaluate its effect on the flow around the graphite sample and its mass loss. An isothermal wall is used in the present computations. For a more accurate analysis, a radiative wall boundary condition will be used. In this work, the FRSC model is used to simulate the constant reaction efficiency approach. In future studies, a more physically viable model will be used for surface reactions where the competing finite rate processes resulting in an effective reaction efficiency for a gas-phase reactant will be accounted for. However, isolating the contributions from different mechanisms is still a challenge. In order to correctly model the mass removal rate, the material response code $\mathrm{MOPAR}^{35}$ will be used in future studies.

\section{Acknowledgments}

The authors gratefully acknowledge funding for this work through Air Force Office of Scientific Research Grant FA-9550-11-1-0309. The authors also acknowledge critical information provided for this research by Professor Doug Fletcher and his graduate students Andrew Lutz and Walt Owens at the University of Vermont.

\section{References}

\footnotetext{
${ }^{1}$ Marschall, J. and Fletcher, D. G., "High-Enthalpy Test Environments, Flow Modeling and In Situ Diagnostics for Characterizing Ultra-High Temperature Ceramics," Journal of the European Ceramic Society, Vol. 30, No. 11, 2010, pp. 2323-
} 
2336.

${ }^{2}$ Laub, B. and Venkatapathy, E., "Thermal Protection System Technology and Facility Needs for Demanding Future Planetary Missions," International Workshop on Planetary Probe Atmospheric Entry and Descent Trajectory Analysis and Science, October 2003.

${ }^{3}$ Gnoffo, P. A., "Planetary-Entry Gas Dynamics," Vol. 31, 1999, pp. 459-94.

${ }^{4}$ Barbante, P., "Heat Flux Duplication Between Ground Facility and Hypersonic Flight," Journal of Thermophysics and Heat Transfer, Vol. 23, No. 4, October-December 2009, pp. 684-692.

${ }^{5}$ Wright, M. J., Grinstead, J. H., and Bose, D., "A Risk-Based Approach for Aerothermal/ TPS Analysis and Testing," Experiment, Experiment, Modeling and Simulation of Gas-Surface Interactions for Reactive Flows in Hypersonic Flights, Vol. RTO-EN-AVT-142, 2007.

${ }^{6}$ Lutz, A., Owens, W., Meyers, J., Fletcher, D., and Marschall, J., "Investigation of CN Production from Carbon Materials in Nitrogen Plasmas," AIAA Paper 2011-901, January 2011.

${ }^{7}$ Owens, W. P., Uhl, J., Dougherty, M., Lutz, A., Meyers, J., and Fletcher, D. G., "Development of a 30kW Inductively Coupled Plasma Torch for Aerospace Material Testing," AIAA Paper 2010-4322, June 2010.

${ }^{8}$ Anna, A. and Boyd, I. D., "Computation of surface catalysis for graphite exposed to high-enthalpy nitrogen flow," $A I A A$ Paper 2012-534, January 2012.

${ }^{9}$ Anna, A., Boyd, I. D., Colombo, V., Ghedini, E., Sanibondi, P., Boselli, M., and Gherardi, M., "Computational modeling of surface catalysis for graphite exposed to high-enthalpy nitrogen flow," RTO-AVT-199, January 2012.

${ }^{10}$ Scalabrin, L. C. and Boyd, I. D., "Numerical Simulation of Weakly Ionized Hypersonic Flow for Reentry Configurations," AIAA Paper 2006-3773, June 2006.

${ }^{11}$ Scalabrin, L. C. and Boyd, I. D., "Numerical Simulations of the FIRE-II Convective and Radiative Heating Rates," AIAA Paper 2007-4044, June 2007.

${ }^{12}$ Martin, A., Scalabrin, L., and Boyd, I., "High Performance Modeling of Atmospheric Re-entry Vehicles," Journal of Physics: Conference Series, Vol. 341, 2012, Article 012002.

${ }^{13}$ MacCormack, R. and Candler, G., "The Solution of the Navier-Stokes Equations Using Gauss-Siedel Line Relaxation," Computers and Fluids, Vol. 17, 1989, pp. 135-150.

${ }^{14}$ Karypis, G. and Kumar, V., "METIS: A Software Package for Partitioning Unstructured Graphs, Partitioning Meshes, and Computing Fill-Reducing Orderings of Sparse Matrices," University of Minnesota, 1998.

${ }^{15}$ Marschall, J. and MacLean, M., "Finite-Rate Surface Chemistry Model, I: Formulation and Reaction System Examples," AIAA Paper 2011-3783, June 2011.

${ }^{16}$ MacLean, M., Marschall, J., and Driver, D. M., "Finite-Rate Surface Chemistry Model, II: Coupling to Viscous NavierStokes Code," AIAA Paper 2011-3784, June 2011.

${ }^{17}$ Alkandry, H., Farbar, E. D., and Boyd, I. D., "Evaluation of Finite-Rate Surface Chemistry Models for Simulation of the Stardust Reentry Capsule," AIAA Paper 2012-2874, June 2012.

${ }^{18}$ Rudy, D. H. and Strikwerda, J. C., "Boundary conditions for subsonic compressible navier-stokes calculations," Computers and Fluids, Vol. 9, 1981, pp. 327-338.

${ }^{19}$ Gupta, R., Yos, J., Thompson, R., and Lee, K., "A review of reaction rates and thermodynamic and transport properties for an 11-species air model for chemical and thermal nonequilibrium calculations to 30,000K." Vol. NASA-RP-1232, 1990.

${ }^{20}$ Wilke, C. R., "A Viscosity Equation for Gas Mixtures," 1950.

${ }^{21}$ Blottner, F. G., Johnson, M., and Ellis, M., "Chemically Reacting Viscous Flow Program for Multi-Component Gas Mixtures," Sc-rr-70-754, Sandia Laboratories, Albuquerque, New Mexico, 1971.

${ }^{22}$ Vincenti, W. G. and Kruger, C. H., Introduction to Physical Gas Dynamics, Krieger Publishing Company, 2002.

${ }^{23}$ Gnoffo, P. A., Gupta, R. N., and Shinn, J. L., "Conservation equations and physical models for hypersonic air flows in thermal and chemical nonequilibrium," Nasa-tp-2867, NASA Langley, Hampton, Virginia, 1989.

${ }^{24}$ Park, C., Nonequilibrium Hypersonic Aerothermodynamics, John Wiley \& Sons, 1990.

${ }^{25}$ Sutton, K. and P.A.Gnoffo, "Multi Component Diffusion with application to computational aerothermodynamics," AIAA Paper 1998-2575, 1998.

${ }^{26}$ Lutz, A., Owens, W., Meyers, J., Fletcher, D., and Marschall, J., "Investigation of CN Production from Carbon Materials in Nitrogen Plasmas," AIAA Paper 2011-901, January 2011.

${ }^{27}$ McBride, B. J. and Gordon, S., "Chemical Equilibrium with Applications (CEA)," [http://www.grc.nasa.gov/WWW/CEAWeb/], accessed in 2011.

${ }^{28}$ Singh, H., Coburn, J. W., and Graves, D. B., "Recombination coefficients of O and N radicals on stainless steel," Journal of Applied Physics, Vol. 88, No. 3748, 2000.

${ }^{29}$ Adams, S. F. and Miller, T. A., "Surface and volume loss of atomic nitrogen in a parallel plate rf discharge reactor," Plasma Sources Sci. Technol., Vol. 9, 2000, pp. 1-8.

${ }^{30}$ Gordeev, A. N., Kolesnikov, A. F., and Yakushin, M. I., "Effect of surface catalytic activity on nonequilibrium heat transfer in a subsonic jet of dissociated nitrogen," Fluid Dynamics, Vol. 20, 1985, pp. 478-484.

${ }^{31}$ Driver, D. M. and MacLean, M., "Improved Predictions of PICA Recession in Arc Jet Shear Tests," AIAA Paper 2011-141, January 2011.

${ }^{32}$ Park, C. and Bogdanoff, D. W., "Shock-Tube Measurement of Nitridation Coefficient of Solid Carbon," Journal of Thermophysics and Heat Transfer, Vol. 20, No. 3, July-September 2006.

${ }^{33}$ Pointwise Version 16.04, Pointwise 2011.

${ }^{34}$ Sheppard, R. G., Mathes, D. M., and Bray, D. J., Properties and Characteristics of Graphite, Poco Graphite, Inc., November 2001

${ }^{35}$ Martin, A. and Boyd, I. D., "Simulation of pyrolysis gas within a thermal protection system," AIAA Paper 2008-3805, June 2008. 